\title{
ISING CHAIN IN THE GENERALIZED BOLTZMANN-GIBBS STATISTICS
}

\author{
R.F.S. ANDRADE \\ Instituto de Fisica, Universidade Federal da Bahia, 40210 Salvador, Brazil
}

Received 2 January 1991

The partition function and specific heat of the Ising chain are evaluated in a generalization of the Boltzmann-Gibbs statistics. The extensivity and the influence of the ground state energy in the thermodynamical properties are discussed.

A possible generalization of the Boltzmann-Gibbs statistics (BGS) has been discussed recently by Tsallis [1], who proposed a generalization of the definition of the entropy as

$$
S_{q}=k \frac{1-\sum_{i=1}^{w} p_{i}^{q}}{q-1}
$$

where $i=1, \ldots, w$ counts the different microstates (configurations). This relation stems from the study of multifractals and is related to other entropy definitions of information theory: it is very close to the definition introduced by Daróczy [2], and it is also related with the Rényi entropy of information theory [3] by

$$
\bar{S}_{q} \equiv k \frac{\log \sum_{i=1}^{w} p_{i}^{q}}{1-q}=k \frac{\log \left[1+(1-q) S_{q} / k\right]}{1-q}
$$

In the quoted expressions $q$ is the defining parameter of the statistics, which recovers the usual BG'S in the limit $q \rightarrow 1$.

In this note wi discuss the behaviour of an Ising chain for a family of statistics from the quoted set. The model is a natural many particle extension of the two-level model presented in ref. [1]. We aim to enlighten some points concerning the influence of the ground state on the results. The results we obtained also allow for a discussion on the extensivity of the quantities 
evaluated within these statistics. The choice of the values of $q$ allows for exact results and for a connection to the BGS.

The entropy definition (1) leads to a canonical distribution for a system in thermal bath as

$$
-\frac{\left[1-\beta(q-1) \varepsilon_{i}\right]^{1 /(q-1)}}{Z_{q}}, \quad Z_{q}=\sum_{i=1}^{w}\left[1-\beta(q-1) \varepsilon_{i}\right]^{1 /(q-1)} .
$$

We realize that the family

$$
q_{r}=\frac{r+1}{r}, \quad r=1,2, \ldots,
$$

reduces (3) to a polynomial expression as

$$
p_{r} \sim\left(1-\frac{\beta E}{r}\right)^{r}
$$

With such $p_{r}$ the sums over configurations can be carried out without difficulty for the Ising model. Moreover we note that, for any finite $r$, (5) constitutes a truncation of the exponential function of $-\beta E$. Therefore it is natural to expect that the results given by the finite-r statistics should converge to those of BGS in the high temperature regime.

We will define the Ising Hamiltonian as

$$
H=\bar{E}-J \sum_{i=1}^{N} \sigma_{i} \sigma_{i+1}, \quad \sigma_{i}= \pm 1,
$$

where we explicitly introduced the constant parameter $\bar{E}$. It allows for a calibration of the ground state energy, which strongly influences the behaviour of the system. This becomes clear by the results that we get by choosing two values $\bar{E}_{1}=-N J$ and $\bar{E}_{2}=N J$. In the first case the energy levels are nonpositive, the values going from $-2 N J$ to 0 . In the second case the levels have nonnegative values, from 0 to $2 N J$. These levels are the same as, for instance, the ones of a lattice gas (with site variables 0 or 1 ) with negative (positive) coupling.

Let us analyse the first case. After a $\sigma \tau$ transformation the Hamiltonian (6) is reduced to

$$
H=-N J-J \sum_{i=1}^{N} \tau_{i}, \quad \tau_{i}= \pm 1 .
$$

The partition function $Z_{r}$ for any value of $r$ can be obtained after a little effort. 
Indeed we have

$$
\begin{aligned}
Z_{r} & =\sum_{\text {conf }}\left(1+\frac{N \beta J}{r}+\frac{\beta J}{r} \sum_{i=1}^{N} \tau_{i}\right)^{r} \\
& =\sum_{m=0}^{r} C_{r}(m)\left(1+\frac{N \beta J}{r}\right)^{r-m} \frac{\beta^{m} J^{m}}{r^{m}} F(m, N),
\end{aligned}
$$

where $C_{r}(m)=r ! / m !(r-m) !$ and $F(m, N)=\Sigma_{\text {conf }}\left(\Sigma_{i} \tau_{i}\right)^{m}$ can be evaluated by counting the number of contributions for all possible configurations of positive and negative bonds $\tau_{i}$. We arrive at

$$
F(m, N)=\left.\left(2 P \frac{\partial}{\partial p}-N\right)^{m}(p+q)^{N}\right|_{p=q=1} .
$$

After (9) the definite evaluation of $F(m, N)$ for successive values of $m$ is straightforward, but it becomes increasingly lengthy for large values of $\boldsymbol{m}$. Since the final expressions are power series of $N$, it is convenient to derive a recursion relation for the coefficients, which can be easily implemented in a numerical program. So we first write

$$
\left(2 p \frac{\partial}{\partial p}-N\right)^{m}(p+q)^{N}=(p+q)^{N-m} \sum_{n=0}^{m} N^{n} \sum_{s=0}^{m} \eta_{n s}^{m} p^{s} q^{m-s},
$$

where $\eta_{n s}^{m}(m, n, s \geqslant 0)$ are recursively given by

$$
\eta_{n s}^{m+1}=\eta_{n-1, s-1}^{m}+(s-m-1) \eta_{n, s-1}^{m}+s \eta_{n s}^{m}
$$

with initial conditions $\eta_{n s}^{0}=\delta_{n, 0} \delta_{s, 0}$. From these $\eta$ 's we define

$$
\gamma_{m n}=\sum_{s=0}^{m} \eta_{n s}^{m}, \quad \zeta_{m u}=\sum_{n=0}^{u} C_{m}(n) \gamma_{m-n . u-n}(-1)^{n}
$$

to finally write $F(m, N)$ as

$$
F(m, N)=2^{N} \sum_{u=0}^{m} \zeta_{m u} N^{u}
$$

The evaluation of the $\zeta$ 's according to the above prescription leads to $\zeta_{m u} \equiv 0$ for $m$ odd, $\forall u$, as could be anticipated. We also find $\zeta_{m u} \equiv 0$ for $m$ even, $u>\frac{1}{2} m$. Just to illustrate we quote some values of $\zeta_{m u}: \zeta_{2.1}=1 ; \zeta_{4.1}=-2$; $\zeta_{4,2}=3 ; \zeta_{6,1}=16 ; \zeta_{6,2}=-30 ; \zeta_{6,3}=15$ and so on.

Finally insering (13) into (8) leads to 


$$
Z_{r}=2^{N} \sum_{m=0}^{r} \sum_{s=0}^{r-m}\left(\frac{\beta J}{r}\right)^{r-s} \frac{r !}{s ! m !(r-m-s) !} \sum_{u=0}^{m} \zeta_{m u} N^{r+u-m-s}
$$

The connection between the generalized partition function (14) and thermodynamics is still an open question. Therefore the way to analyse the properties of the model follows the evaluation of its mean energy $U_{r}$, defined as

$$
U_{r}=\frac{1}{Z_{r}} \sum_{\text {conf }}\left(-N J-J \sum_{j=1}^{N} \tau_{j}\right)\left(1+\frac{N \beta J}{r}+\frac{\beta J}{r} \sum_{i=1}^{N} \tau_{i}\right)^{r}
$$

The evaluation of (15) follows the same steps as for $Z_{r}$, and we finally get

$$
U_{r}=-N J-J \frac{\sum_{m=0}^{r} \sum_{s=0}^{r-m}\left(\frac{\beta J}{r}\right)^{r-s} \frac{r !}{s ! m !(r-m-s) !} \sum_{u=0}^{m+1} \zeta_{m+1, u} N^{r+u-m-s}}{\sum_{m=0}^{r} \sum_{s=0}^{r-m}\left(\frac{\beta J}{r}\right)^{r-s} \frac{r !}{s ! m !(r-m-s) !} \sum_{u=0}^{m} \zeta_{m u} N^{r+u-m-s}}
$$

The positive analysis of the second case $(\bar{E}=N J)$ is more difficult, due to the fact that the polynomial expression (5) may cause some positive levels to have a negative probability when $r$ is odd. Such levels ought to be cut off in order that the expression (5) have some meaning. We will multiply the expression (6) by the Heaviside function $\theta(1-\beta E)$ to be sure that only those levels with energy $E \leqslant E^{\prime}=r / \beta$ make contributions to $Z_{r}$. When $r$ is even the probabilities will also be positive when $E>E^{\prime}$. Nevertheless we will keep the factor $\theta(1-\beta E)$ in order to have similar results for any value of $r$, and the partition function will have the following form:

$$
Z_{r}=\sum_{\text {conf }}\left(1-\frac{N \beta J}{r}+\frac{\beta J}{r} \sum_{i=1}^{N} \tau_{i}\right)^{r} \theta\left(1-\frac{N \beta J}{r}+\frac{\beta J}{r} \sum_{i=1}^{N} \tau_{i}\right)
$$

The presence of the Heaviside function complicates the derivation of an analytic expression for $Z_{r}$, since it takes off several terms of the sum over configurations. Moreover the number of terms left out is a function of the temperature, so that possible analytical expressions would be restricted to certain temperature ranges.

Since we may identify

$$
\sum_{\text {conf }} \sum_{i} \tau_{i} \rightarrow \sum_{M=0}^{N} C_{N}(M)(N-2 M)
$$


the most convenient way to write $Z_{r}$ of the positive energy levels is to introduce $\bar{N}_{r}(T)=r k_{\mathrm{B}} T / 2 J$ to obtain

$$
Z_{r}=\sum_{M=0}^{\bar{N}_{r}} C_{N}(M)\left(1-\frac{2 M \beta J}{r}\right)^{r}
$$

which as function of $T$ can be obtained by numerically summing over the allowed energy levels. The internal energy $U_{r}$ will also be obtained numerically from the expressions

$$
U_{r}=\frac{1}{Z_{r}} \sum_{M=0}^{\bar{N}_{r}} C_{N}(M)\left(1-\frac{2 M \beta J}{r}\right)^{r} 2 J M .
$$

From the expressions (16) and (20) we may also obtain the specific heat of the model with two ground state energies in this family of statistics as

$$
c_{r}=-\frac{1}{N k_{\mathrm{B}} T^{2}} \frac{\partial U_{r}}{\partial \beta}
$$

Let us begin with the negative energy levels (16). Since the general expression is very cumbersome we write down only the expressions for the first two values of $r$ :

$$
\begin{aligned}
& \frac{c_{1}}{k}=\frac{J^{2}}{(k T+N J)^{2}}, \\
& \frac{c_{2}}{k}=4 J^{2} \frac{4 k^{2} T^{2}+4 k T N J+J^{2} N(N-1)}{\left[4 k^{2} T^{2}+4 k T N J+J^{2} N(N+1)\right]^{2}} .
\end{aligned}
$$

These expressions deviate from the expected behaviour for intensive quintities in the BGS: both are proportional to $1 / N^{2}$ in the limit of large number of spins. This is illustrated in figs. 1a and $\mathrm{ib}$, where we draw $c_{1}$ and $c_{2}$ as functions of the reduced temperature $k_{\mathrm{B}} T / J$ for several numbers of spins. The curves also show clearly that $c_{r} \rightarrow 0$ as $T \rightarrow \infty$ for any number of spins. The unexpected vanishing of $c_{r}(r=1,2)$ in the thermodynamic limit may be discussed along two lires: The first one concerns the expression of the energy, which is indeed an extensive quantity. However, in the limit $N \rightarrow \infty, U$, approaches a constant value, which causes the specific heat to vanish. The second way to look at this result is to recall that the finite-r statistics is to a polynomial truncation of the exponential function, so that the results should approach those of BGS when $\beta E$ is small. When $N$ increases, so do the values of the energy levels which make contributions to physical properties. Therefore $\beta E$ will be small for 

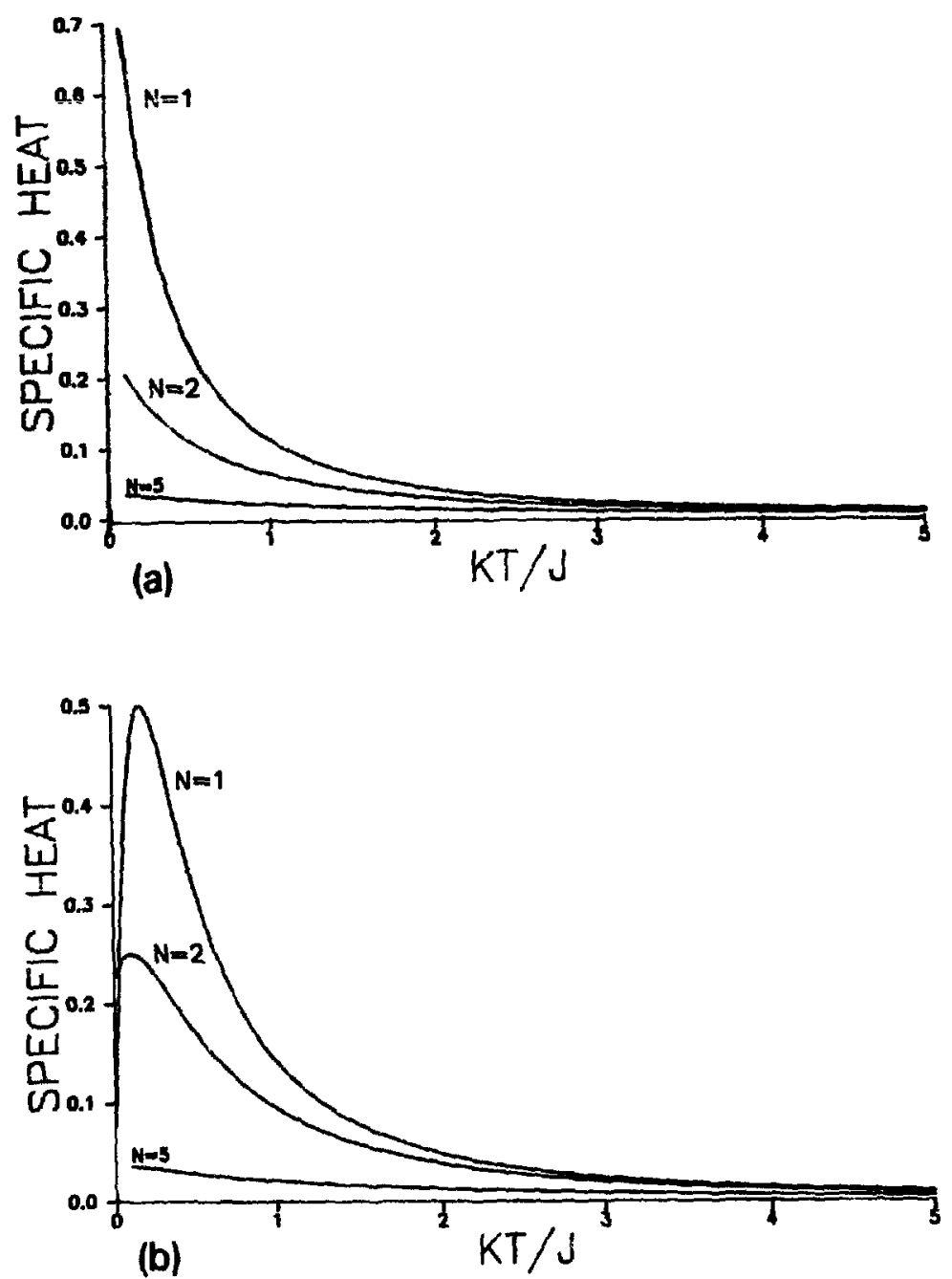

Fig. 1. Specific heat of the Ising chain with negative energy levels when $r=1$ (a) and $r=2$ (b), for different numbers of spins in the chain. It converges uniformly to zero as $n$ grows.

larger values of the temperature. Thus when we are not close to the region where $\beta E$ is small $(N \rightarrow \infty$ or $T \rightarrow 0)$ the results will deviate from those of the BGS.

Analytical expressions for the specific heat in the case where the levels are positive cannot be obtained. Therefore we are forced to derive (20) and evaluate numerically the sums appearing in the expressions. The results so obtained are plotted in figs. $2 \mathrm{a}(r=1)$ and $2 \mathrm{~b}(r=2)$. We observe an entirely different picture with respect to the former results. The specific heat now presents oscillations with respect to the temperature, which are sharp for $r=1$ and somewhat smoother for $r=2$. Such behaviour is not common to known systems, bur is like the oscillations observed for the specific heat of one harmonic oscillator within the same statistics [4]. Their origin is related io the sudden occupation of some energy levels when a small variation of the 

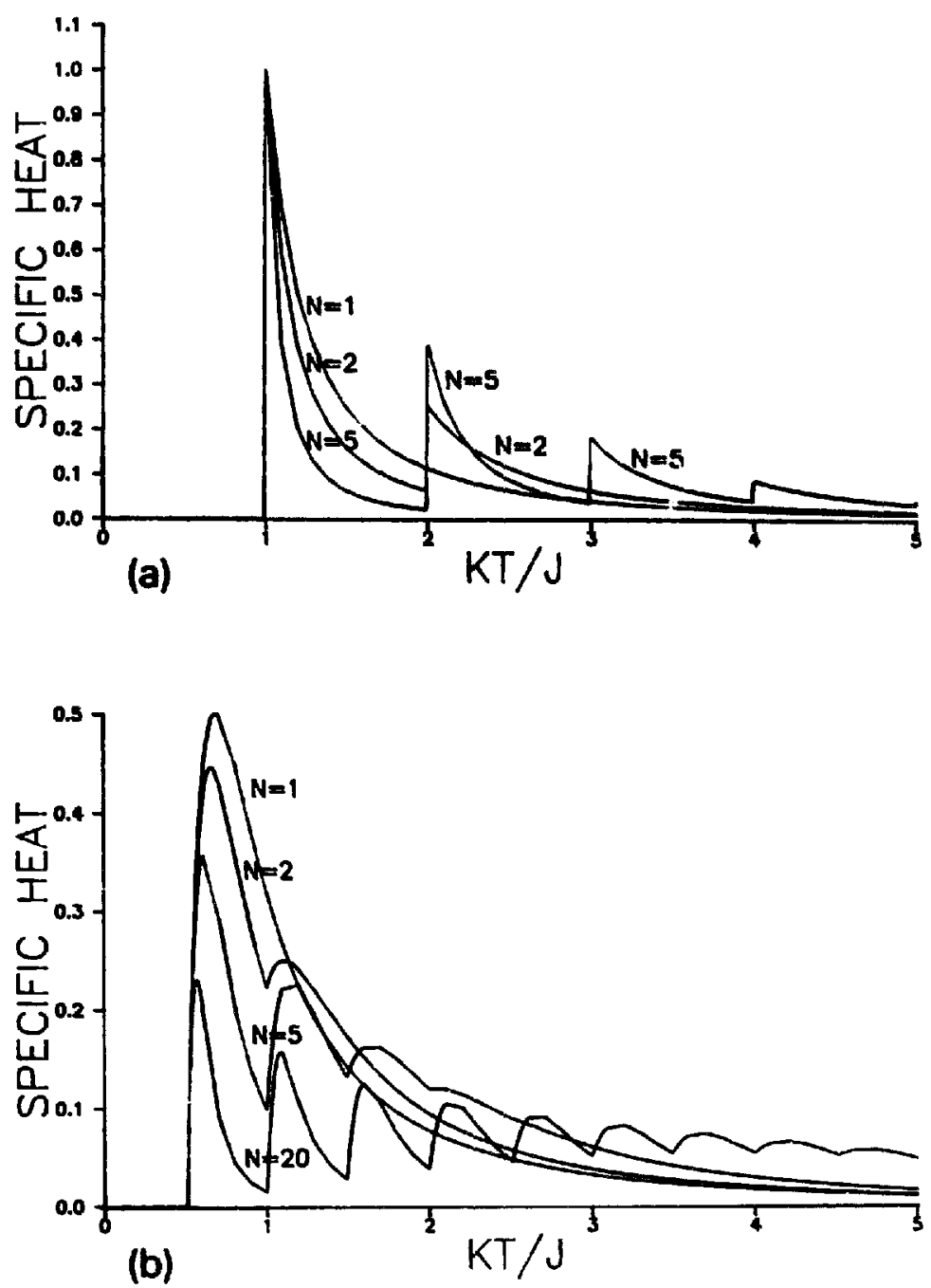

Fig. 2. Specific heat of the Ising chain with positive energy levels when $r=1$ (a) and $r=2$ (b). for different numbers of spins in the chain. Note the presence of oscillations due to the population of new levels as the temperature is increased. The interval between the peaks is $k T / J=1 / r$. The number of peaks increases with $N$, since there exists a larger number of levels which will only be populated at higher temperatures.

temperature allows for an increased value of $\bar{N}_{r}$. One interesting feature displayed by fig. $2 \mathrm{~b}$ refers to the behaviour of $c_{2}$ when $N$ increases. We note that when $N=20$ the value of $c_{2}$ is larger than the ones for $N=1,2$ and 5 as $T$ increases. Such increase of the high temperature $c_{2}$ with $N$ stays in contrast with the monotonic decrease of $c_{r}$ with $N$ when the levels are negative. The same situation is shown in fig. $2 \mathrm{a}$, though not so clear as in fig. $2 \mathrm{~b}$. We may observe, for instance, that the $N=2$ curve lies below the $N=1$ until $k T / J=2$, when the $N=2$ curve (but not the $N=1$ ) has a peak making its value higher than the $N=1$ curve. For values $k T / J>2$ the $N=2$ curve will be higher than that of $N=1$. The same situation is observed with the other values of $N$. 
Due to the complex oscillatory behaviour just discussed and to the lack of analytical expressions it is rather difficult to make a definite discussion on the behaviour of the specfic heat in the limit $N \rightarrow \infty$, when the energy levels are positive. Nevertheless, the results obtained for $N$ as high as 100 suggest that it will converge to zero for any value of $T$, though the convergence seems to be nonuniform.

The results just discussed illustrate the very interesting behaviour of the simple Ising chain in the generalized statistics. The series of statistics we chose converges to the usual GBS in the limit $r \rightarrow \infty$, and the results obtained for finite $r$ are equivalent to those of BGS in the high temperature limit. A natural conjecture refers to a connection between the high temperature expansions and the finite-r statistics. The other important feature is the influence of the ground state energy. Our results point out that if all levels are allowed for any value of the temperature, the link with the usual GBS is more naturai. On the other hand, if new levels are allowed when the temperature is changed, some unusual behaviour such as the oscillation in the specific heat will be present.

We finally point out that the procedure followed in this letter may be extended to higher dimensions and other known models of statistical mechanics. We may quote, for instance, that the Ising model on the two-dimensional square lattice may be easily evaluated for small values of $r$. However, it has not been possible to derive a general expression such as the one presented in (9).

\section{References}

[1] C. Tsallis, J. Stat. Phys. 52 (1988) 497.

[2] Z. Daróczy, Inf. Control. 16 (1970) 36.

[3] A. Rényi, Probability Theory (North Holland, Amsterdam, 1970).

[4] N. Ito and C. Tsallis, Nuovo Cimento 11D (1989) 907. 